\title{
788 A NOVEL T- LYMPHOCYTE BINDING APTAMER ASSEMBLED INTO A BISPECIFC COMPOUND FOR THE TREATMENT OF SOLID TUMORS
}

Irit Carmi Levy*, Erez Lavi, Neta Zilony Hanin, Zohar Pode, Karin Mizrahi, Ronit Farhi, Anastasia Paz, Neria Reiss, Chen Shimoni, Vitaliy Buravenkov, Liron Levy-Efrati, Rotem Haimovich, Ohad Glaich, Itay Liron, Hadas Aharoni Zazrin, Ido Bachelet, Raanan Berger, Guy Neev. Aummune, Tel Aviv, Israel

Background T-cell engagers are bispecific molecules directed against the CD3 complex on one end and a tumor specific antigen on the other end, allowing a physical link of $\mathrm{T}$ cell to a tumor cell, resulting in tumor killing and immune activation. Bispecific molecules harnessing and redirecting T-cells towards tumor cells are a promising therapeutic agents. Aptamers are single stranded oligonucleotides with binding and recognition propensities similar to those of antibodies. Aptamers have a number of advantages over bispecific antibodies including shorter generation time and low immunogenicity. Thus, aptamers capable of targeting $\mathrm{T}$ cells would have great potential for use as anti-cancer therapeutics

Methods Systematic evolution of ligands by exponential enrichment (SELEX) methodology was employed in order to identify a novel CD3e binding aptamer. CD3 binding aptamer was subsequently linked into a bispecific $\mathrm{T}$ cell engager structure with a tumor-targeting aptameric arm. The tumor-targeting aptamer is developed by Aummune's proprietary tailored therapeutic platform. ${ }^{1}$ based on identifying functional aptamer sequences capable of specifically killing targeted tumor cells and sparing healthy tissue .Exemplary bispecific aptamers were tested for $\mathrm{T}$ cell stimulation by flow cytometry. In vivo antitumor activity was investigated in syngeneic and in xenograft tumor models.

Results We have successfully identified a novel CD3e -targeting aptamer with a $\mathrm{Kd}$ of $31 \mathrm{nM}$. A bispecific $\mathrm{T}$ cell engager comprised of this aptamer and a tumor-targeting aptamer induced a potent stimulation of $\mathrm{T}$ cells in vitro, resulting in CD69 upregulation and IFNg secretion.Next, the CD3e targeting aptamer was hybridized to tumoricidal aptamers identified by Aummune's platform (VS12) to target either the human colon carcinoma HCT116 cells or (VS32) the murine triple negative breast cancer 4T1 cells. Both bispecific entities (CS6VS12 and CS6-VS32) effectively lead to inhibition of tumor growth in vivo and increased survival in the corresponding models.

Conclusions Our data above provide a proof-of-concept for Aummune's Bispecific Aptamer efficacy and provide a framework for the clinical development of this novel tailored immune therapeutic agents. Indeed, we are currently in the process of developing a first-in-human clinical study in subjects with solid tumors.

\section{REFERENCE}

1. Mamet N, et al, Commun Biol 2020.

http://dx.doi.org/10.1136/jitc-2021-SITC2021.788 